\title{
Quantifying Adsorption on Single Alumina Particles via Impact Voltammetry and Current Transient Analysis
}

\author{
Qianqi Lin and Richard G. Compton* \\ Department of Chemistry, Physical and Theoretical Chemistry Laboratory, Oxford University, South \\ Parks Road, Oxford OX1 3QZ, U.K. \\ *Email: richard.compton@chem.ox.ac.uk \\ Phone: +44 (0) 1865275957 \\ Fax: +44 (0) 1865275410
}

KEYWORDS

Aluminium oxide, alumina, catechol, adsorption, particle impacts, surface diffusion on a sphere 


\section{ABSTRACT}

Particle impacts are used to quantify the adsorption of catechol on single alumina particles. In these experiments particles suspended in solution impact a microelectrode held at a suitable potential for the oxidation or reduction of the adsorbed species and the resulting current/time transients "spikes" associated with individual impacts measured. Using theory for charge diffusion over the surface of a sphere, the individual impact spikes arising from the electro-oxidation of adsorbed catechol can be modelled to derive the diffusion coefficient of charge transfer over the surface of alumina as (2.5 \pm $0.5) \times 10^{-6} \mathrm{~cm}^{2} \mathrm{~s}^{-1}$. The coverage of catechol on the surface of alumina is found to be $(5.9 \pm 1.9) \times 10^{-}$ ${ }^{10} \mathrm{~mol} \mathrm{~cm}^{-2}$.

\section{INTRODUCTION}

Particle impact experiments have been introduced as an electrochemical method to detect nanoparticles. When nanoparticles collide with a microelectrode held at a certain oxidising or reducing potential, dissolutive redox reaction of the nanoparticles takes place, giving rise to current "spikes" in chronoamperometry. Measurement of the charge passed in the spikes can be used to determine the size of nanoparticles. However, the use of impact experiments is largely hitherto restricted to conductive and/or redox active nanoparticles, such as silver ${ }^{1}$, gold ${ }^{2}$, nickel ${ }^{3-4}$, copper $^{5}$ and magnetite $\left(\mathrm{Fe}_{3} \mathrm{O}_{4}\right)^{6}$. Nevertheless Quinn, van’t Hof and Lemay ${ }^{7}$ found that insulating particles could be detected via chronoamperometry; the method used is indirect and the particles block the electron transfer between a diffusing redox active species and the electrode. A method for the more direct detection of insulating particles was achieved by non-Faradaic (capacitative) processes ${ }^{8-9}$ and used to measure the potential of zero charge (PZC) of the electrode/electrolyte system. Scholz et al. ${ }^{10}$ applied capacitative impacts to detect the adhesion of single clay particles, allowing the determination of surface charge density of the adhering particle surface. Capacitative impacts were further exploited to study the mechanism of adhesion and spreading of liposomes. ${ }^{11-13}$ 
<smiles>Oc1ccccc1O</smiles>

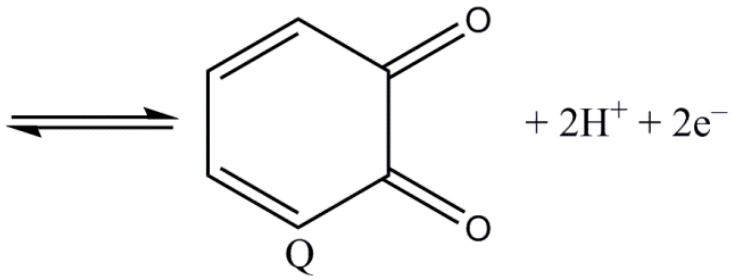

Scheme 1. Two proton, two electron oxidation of catechol $\left(\mathrm{H}_{2} \mathrm{C}\right)$ to the $o$-benzoquinone $(\mathrm{Q})$.

The oxidation of catechol to o-benzoquinone (Scheme 1) at carbon electrodes is catalysed by the presence of alumina particles immobilised on the electrode surface. ${ }^{14}$ The cause of the apparent catalysis for this two-electron, two-proton process is first related to strong adsorption of catechol on the alumina surface. Second, the semiquinone $(\mathrm{SH})$ intermediate formed during reaction can be stabilised by stronger adsorption on the surface of alumina than the parent catechol or the resulting $o$-benzoquinone, both of which are also adsorbed on alumina. Such stabilisation is important since it has been shown that the mechanism of catechol oxidation occurs in solution via the scheme

$$
\begin{aligned}
& \mathrm{H}_{2} \mathrm{C}-\mathrm{e}^{-}-\mathrm{H}^{+} \rightleftarrows \quad \mathrm{SH} \\
& 2 \mathrm{SH} \rightarrow \mathrm{H}_{2} \mathrm{C}+\mathrm{Q}
\end{aligned}
$$

and that the process shows "potential inversion" whereby the oxidation of $\mathrm{H}_{2} \mathrm{C}$ to $\mathrm{SH}$ takes place at a more positive potential than the oxidation of SH to Q. ${ }^{14}$

Building on the understanding of the oxidation of catechol catalysed by alumina-modified electrode surfaces, in this paper a new electrochemistry method is developed furthering experiments with conductive nanoparticles ${ }^{15-16}$ for quantifying adsorption of redox active species on the surface of individual insulating particles. The
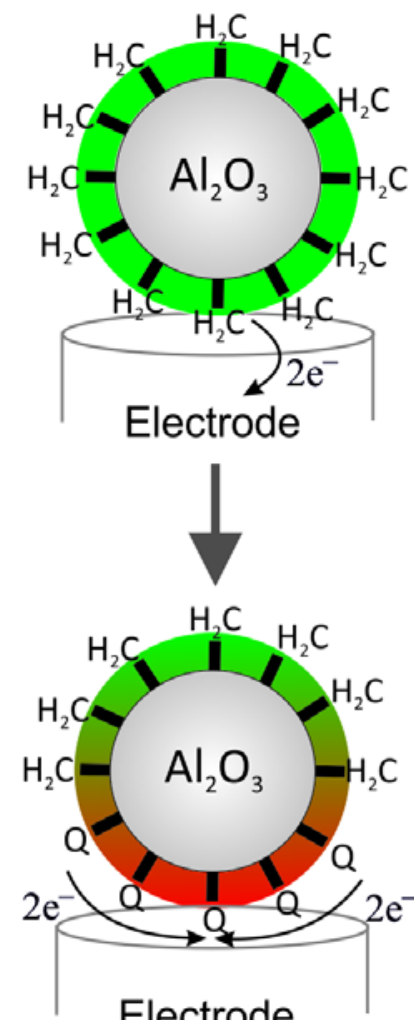

Electrode

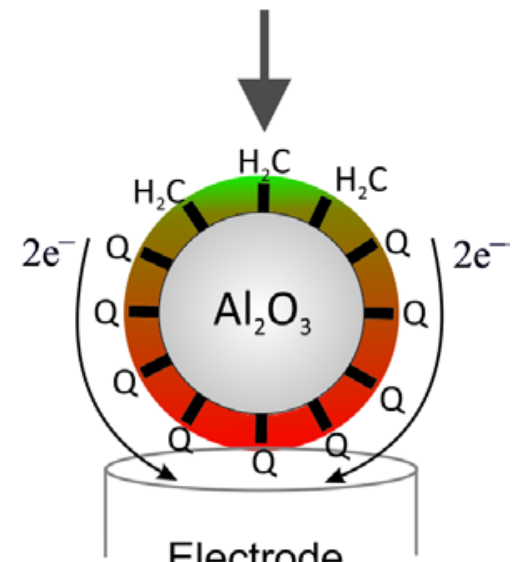

Electrode

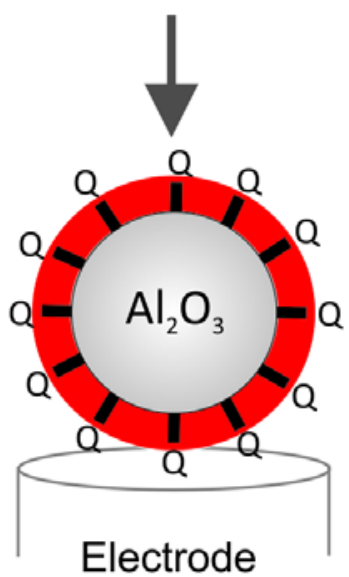

Scheme 2. The model of charge diffusion over the surface of a sphere. $\mathrm{H}_{2} \mathrm{C}$ is catechol and $\mathrm{Q}$ is $o^{-}$ benzoquinone. 
particles, of $c a$. micron size, with surface adsorbed redox active molecules, are suspended in aqueous solution and undergo impacts with a potentiostatted microelectrode. Upon particle-electrode collisions, impact spikes will arise if the electrode is held at a potential which the molecules are reduced or oxidised. The particle cores remain unchanged.

In this new method the shape and size of individual current/time impacts are modelled using theory for diffusion of charge over the surface of the particles as electrons hop from one molecule to another (Scheme 2) ${ }^{17-18}$. Thompson et al. ${ }^{17}$ reported the simulation of the chronoamperometry of electrolysis of species adsorbed on a perfectly spherical particle assuming charge transfer to a substrate electrode at a point contact of negligible resistance. In the present work, the transients from individual impacts, especially the curve shapes, are fitted in the light of this theoretical model. Two parameters, the diffusion coefficient $(D)$ of charge transfer between catechol molecules on the surface of alumina and the coverage $\left(\Gamma_{\mathrm{i}}\right)$ of catechol on the surface of alumina, are extracted.

For the first time, adsorption of redox active species on individual insulating particles will be quantified electrochemically by using impact experiments. Modelling of individual impact spikes will be shown to be in line with the theory for charge diffusion over the surface of a sphere.

\section{THEORY}

In this section we review the theory for Fickian charge diffusion over the surface of a sphere. The perfect sphere model ${ }^{17}$ assumes a full sphere (of radius of $r_{\mathrm{s}}$, Scheme 3), with redox active species attached on the surface, is in contact with an electrode surface at an angle $(\theta)$ of $0^{\circ}$. This is the only contact point where charge transfer occurs and the electrode acts solely to transfer electrons in or out of the particle surface. The changing coverage $\left(\Gamma(\theta) / \mathrm{mol} \mathrm{cm}^{-2}\right)$ of redox active species on the sphere surface during electrolysis can be normalised with respect to the initial coverage $\left(\Gamma_{\mathrm{i}} / \mathrm{mol} \mathrm{cm}^{-2}\right)$. The resulting dimensionless coverage is



Scheme 3 The perfect sphere model with spherical coordinates $(r, \theta$ and $\varphi)$ and the point of contact between the sphere and electrode surface. denoted as $\Gamma(\theta) / \Gamma_{\mathrm{i}}$. The boundary condition for chronoamperometry is that when $\mathrm{t}<0, \Gamma(\theta) / \Gamma_{\mathrm{i}}=1$ everywhere on the sphere; when $\mathrm{t}>0, \Gamma(\theta) / \Gamma_{\mathrm{i}}=0$ at $\theta=0$. 
The assumption is made that the movement of charge obeys Fick's Second Law,

$\frac{\partial \Gamma(\theta)}{\partial t}=D \nabla^{2} \Gamma(\theta)$

where the Laplacian operator in spherical coordinates $(r, \theta, \varphi)$ is defined as

$\nabla^{2}=\frac{\partial^{2}}{\partial r^{2}}+\frac{2 \partial}{r \partial r}+\frac{1}{r^{2}} \frac{\partial^{2}}{\partial \theta^{2}}+\frac{\cos \theta}{r^{2} \sin \theta} \frac{\partial}{\partial \theta}+\frac{1}{r^{2} \sin ^{2} \theta} \frac{\partial^{2}}{\partial \varphi^{2}}$

Since the charge movement is confined to the surface layer as shown in Scheme 2, and as charge transfer only occurs at the point of contact ( $r=r_{\mathrm{s}}$, where $r_{\mathrm{s}}$ is the radius of the sphere), Eq. 4 can be simplified as

$\nabla^{2}=\frac{1}{r_{\mathrm{s}}^{2}} \frac{\partial^{2}}{\partial \theta^{2}}+\frac{1}{r_{\mathrm{s}}^{2} \tan \theta} \frac{\partial}{\partial \theta}$

The equation describing diffusion over the sphere surface can be expressed in dimensionless variables

$\frac{\partial \Gamma(\theta) / \Gamma_{\mathrm{i}}}{\partial \tau}=\frac{\partial^{2} \Gamma(\theta) / \Gamma_{\mathrm{i}}}{\partial \theta^{2}}+\frac{1}{\tan \theta} \frac{\partial \Gamma(\theta) / \Gamma_{\mathrm{i}}}{\partial \theta}$

with the dimensionless time $(\tau)$ defined as

$\tau=t D / r_{\mathrm{s}}^{2}$

where $t$ is time and $D$ is diffusion coefficient of charge transfer over the surface of the sphere. Eq. 6 can be solved numerically based on the boundary condition discussed above using an implicit finite difference method to get the dimensionless coverage $\left(\Gamma(\theta) / \Gamma_{\mathrm{i}}\right)$. The solution can be exploited to derive the expression for charge $(Q)$ at time $t$,

$\frac{Q}{F}=\int_{0}^{\pi} 2 \pi r_{\mathrm{s}}^{2}\left(\Gamma_{i}-\Gamma(\theta)\right) \sin \theta d \theta$

Eq. 8 can be used to derive the current as a function of $t$ for chronoamperometry,

$\frac{I}{F}=\frac{\partial Q}{\partial t}=-2 \pi r_{\mathrm{s}}^{2} \int_{0}^{\pi} \frac{\partial \Gamma(\theta)}{\partial t} \sin \theta d \theta$

or in dimensionless variables as

$$
N u=-\int_{0}^{\pi} \frac{\partial \Gamma(\theta) / \Gamma_{\mathrm{i}}}{\partial \tau} \sin \theta d \theta
$$

with the dimensionless current $(\mathrm{Nu})$ defined as

$N u=\frac{I}{2 \pi \Gamma_{i} D F}$

where $F$ is the Faraday constant $\left(96,485 \mathrm{C} \mathrm{mol}^{-1}\right)$. The plot ("working curve”) of log $\mathrm{Nu} v \mathrm{~s} . \log \tau$ using the perfect sphere model is shown in Figure $1 .{ }^{17}$ 


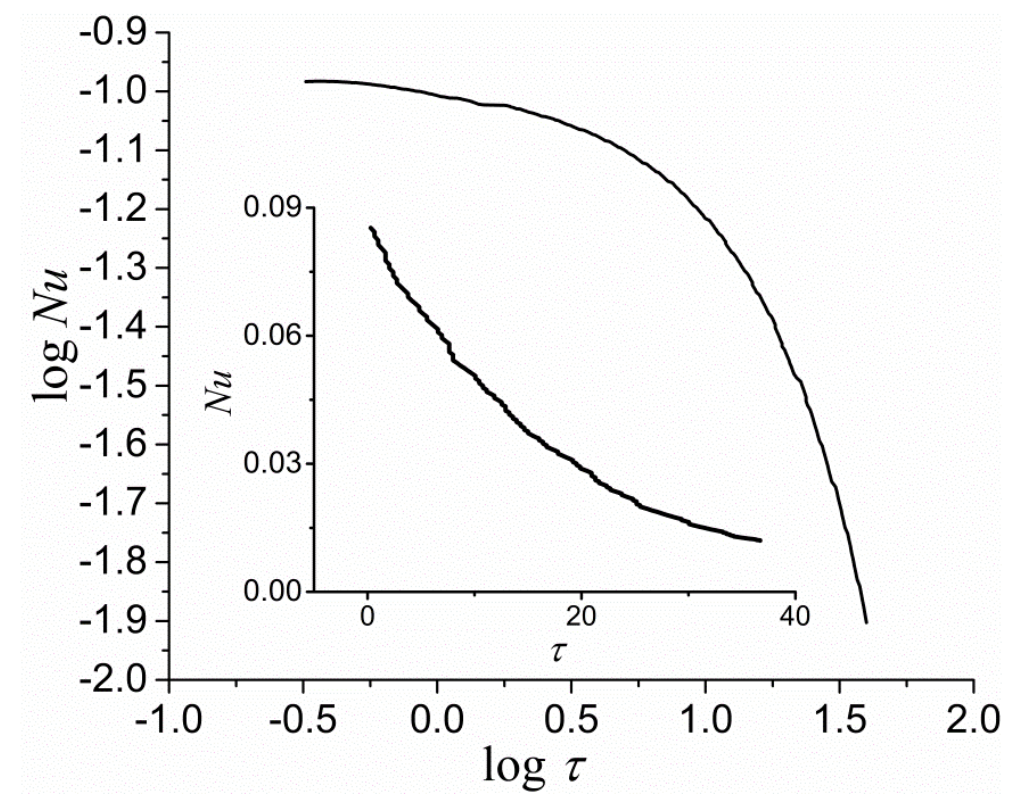

Figure 1. Dimensionless log-log plot for a full sphere in point contact with the electrode surface. Inset: corresponding dimensionless current-time plot for the sphere.

Figure 1 shows that the logarithm of dimensionless current decreases very rapidly at longer times due to exhaustive electrolysis of adsorbate. In the experiments reported below Figure 1 is used for the analysis of individual particle current/time impacts.

\section{EXPERIMENTAL SECTION}

\subsection{Chemical Reagents and Solutions}

Alumina particles were purchased from Buehler (with $80 \sim 100 \%$ “ $1 \mu \mathrm{m}$ ” aluminium oxide). All other chemicals were purchased from Sigma-Aldrich. They were of analytical grade and used without further purification. All solutions were prepared using deionised water of resistivity not less than 18.2 $\mathrm{M} \Omega \mathrm{cm}$ at $298 \mathrm{~K}$ (Millipore, Billerica, MA). pH 3.0 buffered solution was prepared using $0.1 \mathrm{M}$ citric acid/sodium citrate. All solutions were made with $0.1 \mathrm{M} \mathrm{KCl}$ as the supporting electrolyte. $\mathrm{pH}$ buffers were always freshly prepared, measured by using a Hannah pH213 pH meter, and saturated with nitrogen prior to addition of catechol.

\subsection{Apparatus}

Both voltammetric and chronoamperometric measurements were recorded using a $\mu$ AutolabII potentiostat (Autolab, Netherlands) with a standard three-electrode configuration. Either a glassy carbon electrode (GCE, diameter $3.0 \mathrm{~mm}$, ALS distributed by BASi, Tokyo, Japan) or a glassy carbon 
microelectrode ( $\mu$-GCE, diameter $10.0 \mu \mathrm{m}$, Princeton Applied Research, TN, US) was used as the working electrode. A platinum wire (99.99\% GoodFellow, Cambridge, UK) and a saturated calomel electrode (SCE, ALS distributed by BASi, Tokyo, Japan) were used as the counter and reference electrodes, respectively. The working electrodes were polished by using alumina slurry of decreasing particle sizes (1.0-0.05 $\mu \mathrm{m}$, Buehler, IL, US). The electrodes were then sonicated (FB15050, Fisher Scientific, 50/60 Hz, $80 \mathrm{~W}$, Germany) for $1 \mathrm{~min}$ in deionised water and dried by nitrogen. All electrochemical experiments were performed in a three-necked flask with rubber stoppers. The temperature was controlled at $25 \pm 0.2^{\circ} \mathrm{C}$ by a thermostat connecting to the double Faraday cage.

\subsection{Experimental Procedure}

The adsorption of catechol on the alumina was realised in two different ways. For "transfer experiments” at GCEs, $1 \mathrm{~g}$ of alumina was stirred in $25 \mathrm{~mL}$ of $2 \mathrm{mM}$ catechol $\mathrm{pH} 3.0$ buffer supported with $0.1 \mathrm{M} \mathrm{KCl}$. The mixture was stirred and saturated with nitrogen for $3 \mathrm{~h}$, allowing catechol to be fully adsorbed on the alumina. It was left for a further 3 min while the catechol-alumina slurry settled under gravity. This slurry was transferred to a polishing pad for GCE surface modification. After rinsing with water and drying with nitrogen, the electrode was submerged into $25 \mathrm{~mL}$ of blank $\mathrm{pH} 3.0$ buffer supported with $0.1 \mathrm{M} \mathrm{KCl}$ and saturated with nitrogen. Cyclic voltammetry was recorded at a scan rate of $150 \mathrm{mV} \mathrm{s}^{-1}$ in order to observe the redox peak of catechol-alumina.

For impact experiments at $\mu$-GCEs, $0.318 \mathrm{~g}$ of alumina was stirred in $25 \mathrm{~mL}$ of $20 \mathrm{mM}$ catechol pH 3.0 buffer supported with $0.1 \mathrm{M} \mathrm{KCl}$. A higher ratio of catechol:alumina was used in order to maximise the coverage of catechol on alumina. The mixture was stirred and saturated with nitrogen for $3 \mathrm{~h}$. In order to remove the excess catechol, the mixture was centrifuged for 15 min and washed with $\mathrm{pH} 3.0$ buffer. The catechol-alumina particles were transferred to $25 \mathrm{~mL}$ of blank $\mathrm{pH} 3.0$ buffer supported with $0.1 \mathrm{M} \mathrm{KCl}$. By bubbling the solution with nitrogen, the particles dispersed evenly, increasing the frequency of its collision with $\mu$-GCE. Chronoamperometry was recorded for $50 \mathrm{~s}$ at different potentials. The $\mu$-GCE was rinsed with deionised water and dried with nitrogen before each measurement, so as to remove any surface blockage. 


\subsection{Scanning Electron Microscopy Particle Size Characterisation}

The size of catechol-alumina particles was characterised by scanning electron microscope (SEM) imaging. A JEOL JSM-6500F scanning microscope (JEOL GmbH, Eching b. Müchen, Germany) was used with a detector in in-lens geometry. An acceleration voltage of $10.0 \mathrm{kV}$ was applied. Figure 2 shows the size histogram of the 237 particles observed. It is common for such size histograms to follow a log-normal pattern, hence the log-normal distribution was fitted (Figure 2, blue curve) using Origin $\odot$ and the mean radius $\left(r_{\mathrm{s}}\right)$ generated as $0.29 \pm 0.20 \mu \mathrm{m}$.

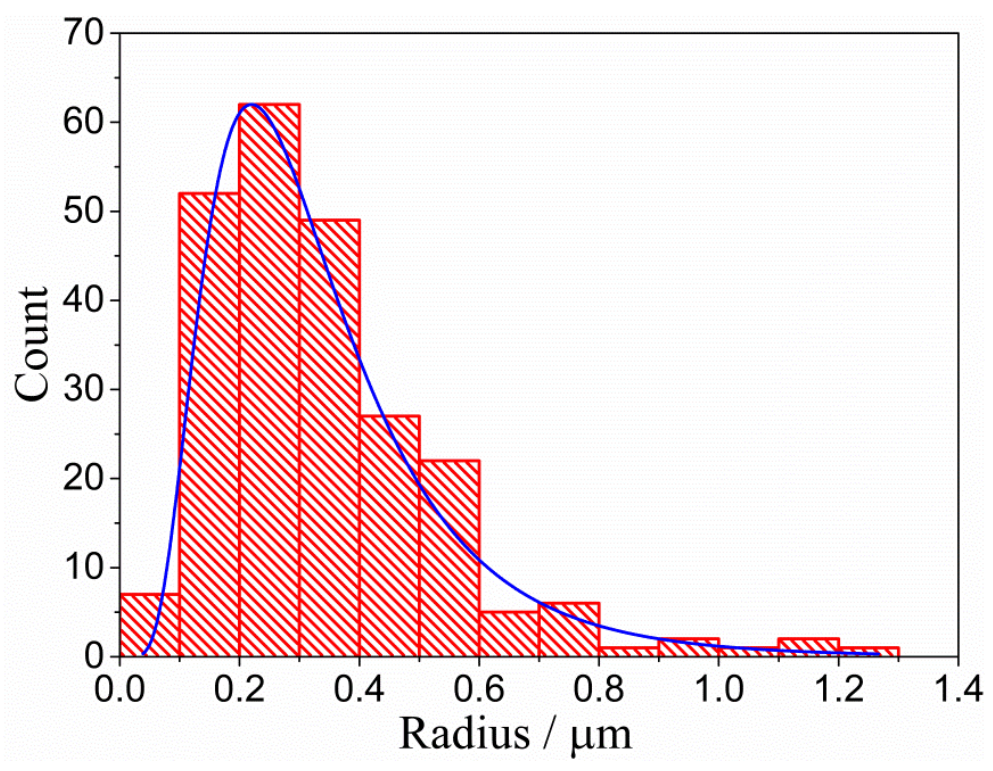

Figure 2. Size histogram of catechol-alumina particles measured from scanning electron microscope image. The log-normal distribution is plotted in the blue curve. The mean radius is $0.29 \pm 0.20 \mu \mathrm{m}$.

\section{RESULTS AND DISCUSSION}

We first report the cyclic voltammetry of ensembles of catechol adsorbed alumina formed via abrasive polishing modification of glassy carbon electrodes. Second, we analyse the chronoamperometry of individual catechol modified alumina particle impacts at glassy carbon microelectrodes. Finally, we fit the impact transients following the model of charge diffusion over the surface of a sphere and measure $D$ and $\Gamma_{\mathrm{i}}$. 


\section{1 “Transfer Experiments” at Glassy Carbon Electrodes}

First, "transfer experiments" were conducted to confirm the voltammetry of catechol adsorbed on alumina at a glassy carbon electrode (GCE) so as to estimate suitable redox potentials for the subsequent impact experiments. Voltammograms were recorded at a catechol-alumina modified GCE in $\mathrm{pH} 3.0$ buffered solution supported with $0.1 \mathrm{M} \mathrm{KCl}$ and saturated with nitrogen (Figure 3, red line). The modified electrode was prepared via abrasive polishing as explained in Section 3. The ensemble voltammetry was run from -0.30 to $+0.90 \mathrm{~V}$ and then reversed to $-0.30 \mathrm{~V}$ at a scan rate of $150 \mathrm{mV} \mathrm{s}^{-1}$. Figure 3 shows that oxidative and reductive peaks can be observed at $c a .+0.42 \mathrm{~V}$ and $+0.35 \mathrm{~V}$, respectively. This is consistent with literature ${ }^{19}$ showing that catechol strongly adsorbed on the surface of alumina undergoes redox conversion to adsorbed o-benzoquinone at these potentials. For comparison, a "blank" voltammogram was recorded in the same solution but on a clean-alumina modified GCE (Figure 3, black line). It is apparent that there is no redox signals observable on the clean-alumina modified GCE. The chemically voltammetry of catechol adsorbed on alumina is fully discussed in the literature ${ }^{19}$.

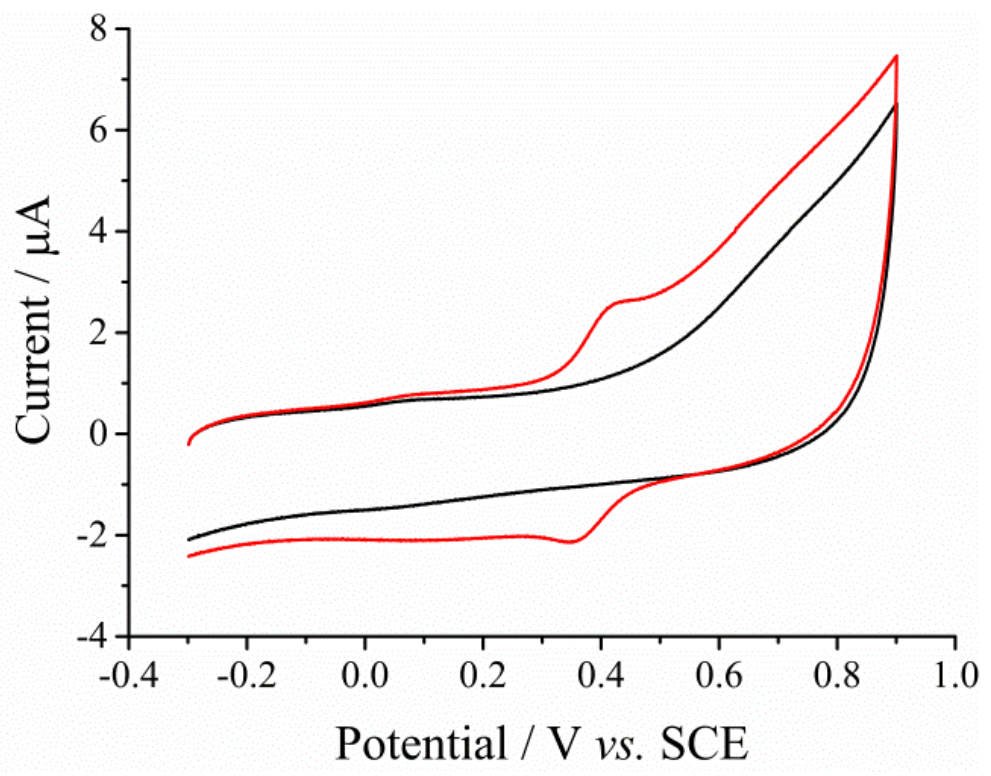

Figure 3. Cyclic voltammograms measured in $\mathrm{pH} 3.0$ buffered solution supported with $0.1 \mathrm{M} \mathrm{KCl}$ and saturated with nitrogen. Scan rate $=150 \mathrm{mV} \mathrm{s}^{-1}$. Red: at a catechol-alumina modified glassy carbon electrode. Black: at a clean-alumina modified glassy carbon electrode. 


\subsection{Impacts at Glassy Carbon Microelectrodes}

According to the results in the preceding section, for the case of catechol-alumina particle impacts at a glassy carbon microelectrode ( $\mu$-GCE), oxidation might be expected to take place when the potential applied to the electrode is at least $c a .+0.40 \mathrm{~V}$ or higher. Chronoamperograms were recorded at the potential of $+0.90 \mathrm{~V}$ after a known amount of catechol-alumina was added. Here a large overpotential was applied to fully drive the exhaustive oxidation of the surface bound catechol (as discussed more fully below). In Figure 4, the red line shows impact spikes corresponding to the oxidation of catechol when the particles are in contact with $\mu$-GCE. The measurements were repeated five times (Not shown). For comparison, chronoamperograms were recorded at $+0.9 \mathrm{~V}$ with addition of the same amount of unmodified alumina. No impact spikes were observed (Figure 4, black line). This confirms that the oxidation of catechol on the surface of alumina is the source of the impact signal. Data for other potentials is reported later.

246 impact spikes were observed over five 50 s scans. The frequency is $c a$. $1 \mathrm{~Hz}$, which is lower than the value estimated based on the Stokes-Einstein Equation and diffusion-limited model.3, ${ }^{20-21}$ This is likely due to near-wall hindered diffusion of the catechol-alumina near the electrodes, ${ }^{22-23}$ and also due to possible partial sedimentation of the alumina suspension.

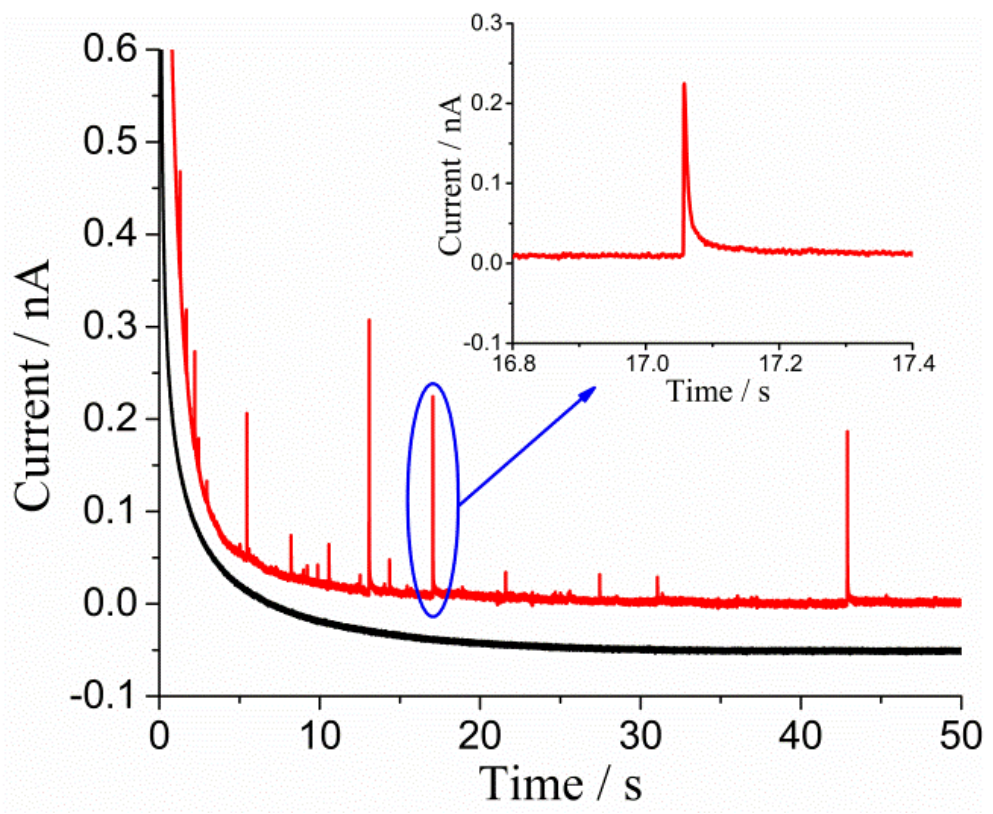

Figure 4. Chronoamperograms measured at $+0.90 \mathrm{~V}$ at a glassy carbon microelectrode. Red: oxidation spikes for catechol-alumina particles. Inset: zoom of circled individual spike. Black: no 
spikes for unmodified alumina particles, with the baseline vertically shifted downwards by $50 \mathrm{pA}$ for clarity.

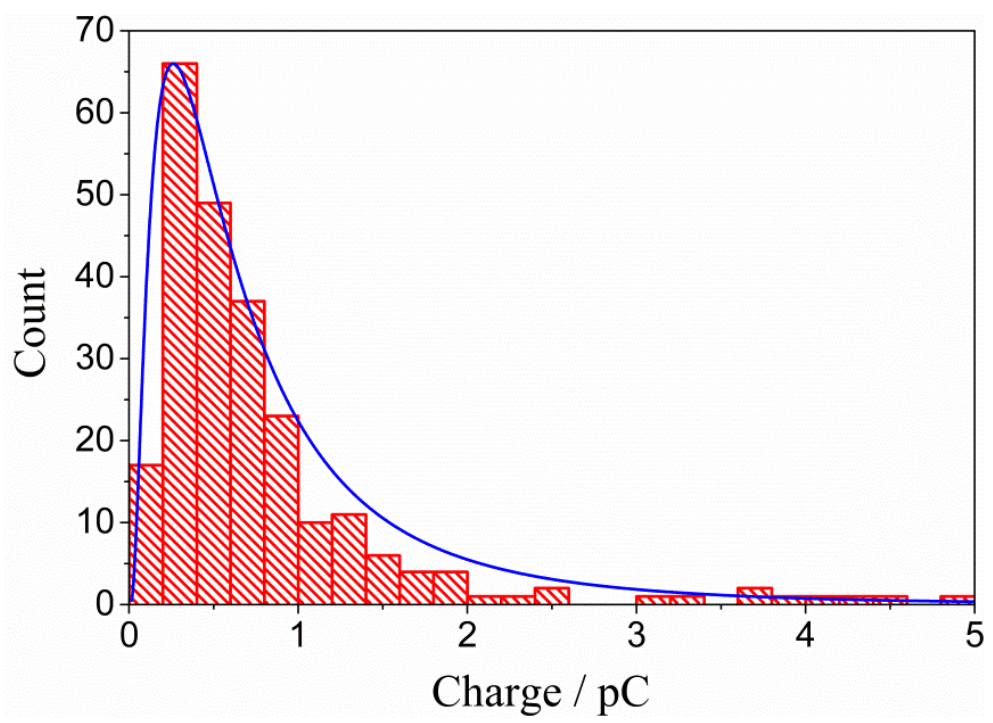

Figure 5. Histogram of charge passed per individual spike, relating to catechol-alumina particles. The log-normal distribution is plotted in the blue curve. The mean charge is $0.60 \pm 0.07 \mathrm{pC}$.

The charge passed during the collision between a catechol-alumina particle and $\mu$-GCE can be derived by integrating the individual current spikes. Figure 5 shows the distribution of charge passed per individual spike (Q) over five 50 s scans. Following the framework of a log-normal distribution, the mean charge is generated by Origin $(c)$ as $0.60 \pm 0.07 \mathrm{pC}$. $Q$ can be related to the coverage of catechol on the alumina particles via

$Q=n e N$

where $n$ is the number of electrons transferred per catechol molecule $(n=2), e$ is the electronic charge and $N$ is the number of catechol molecules adsorbed per alumina particle. The latter is given by the following equation

$N=\frac{S_{\text {alumina }}}{S_{\text {catechol }}}$

where $S_{\text {alumina }}=4 \pi r_{\mathrm{s}}{ }^{2}$ is the surface area of an alumina particle and $S_{\text {catechol }}$ is the molecular area of the adsorbed catechol. Combining Eq. 12 and 13 gives

$S_{\text {catechol }}=\frac{4 \pi r_{\mathrm{s}}^{2} n e}{Q}$ 
where $r_{\mathrm{s}}=0.29 \pm 0.20 \mu \mathrm{m}$ from SEM and $Q=0.60 \pm 0.07 \mathrm{pC}$ from impact experiments. The value of $S_{\text {catechol }}$ can hence be derived as $5.6 \times 10^{-19} \mathrm{~m}^{2}$. This suggests that the coverage is close to that of a full monolayer, since by approximating $S_{\text {catechol }}$ as a rectangle with bond lengths for C-C (aromatic), C-H and C-O being 140, 109 and 136 pm respectively and assuming a close packed array a molecular area of $c a .5 .5 \times 10^{-19} \mathrm{~m}^{2}$ is suggested. With the measured $S_{\text {catechol, }}$, the average coverage $\left(\Gamma_{\mathrm{i}}\right)$ of catechol on the alumina is estimated as $(3.0 \pm 2.5) \times 10^{-10} \mathrm{~mol} \mathrm{~cm}^{-2}$.

It is also helpful to compare the size distribution measured via electrochemical impacts with that measured using SEM combined with the estimated area occupied by an adsorbed catechol molecule on the alumina particle surface. Note that a log-normal distribution is used here, since experimental histograms show the typical pattern of a log-normal distribution. The comparison is shown in Figure 6 and good agreement is seen. The impact data is slightly weighted in favour of smaller, faster diffusing particles and so there is a small discrepancy at larger radii. ${ }^{24}$

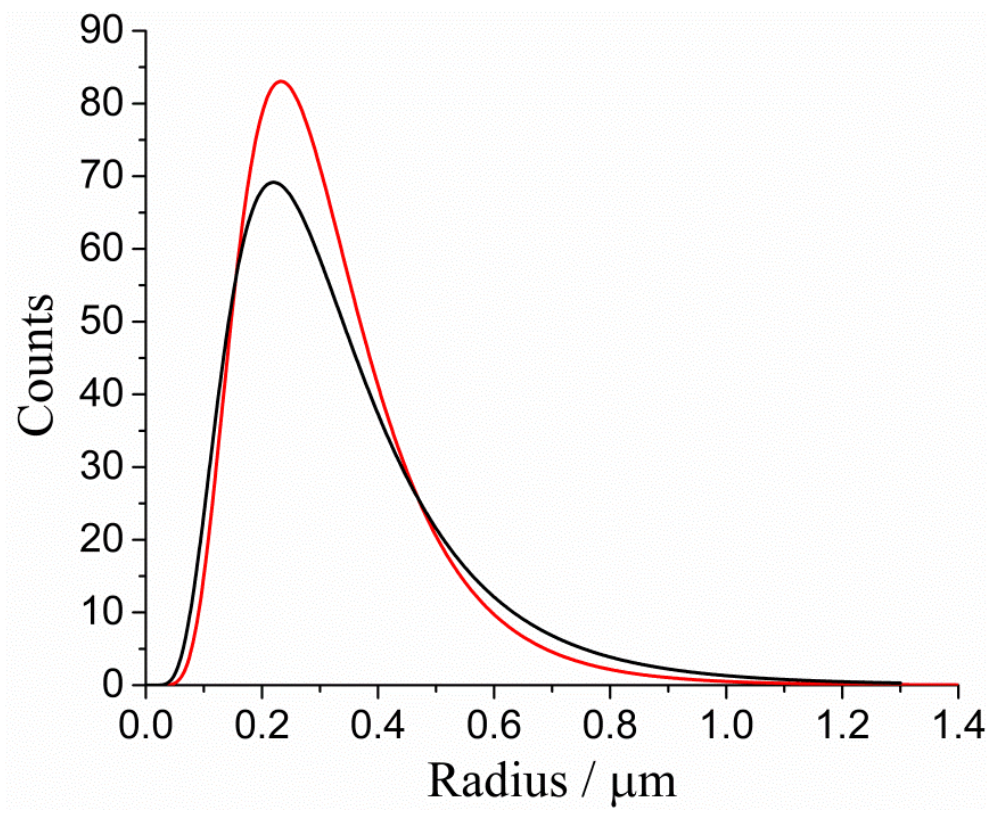

Figure 6. Size distribution of catechol-alumina particles by two methods. Red line: obtained from impacts at a glassy carbon microelectrode. The mean radius is generated from a log-normal distribution as $0.29 \pm 0.15 \mu \mathrm{m}$. Black line: obtained from scanning electron microscope image. The mean radius is generated from a log-normal distribution as $0.29 \pm 0.20 \mu \mathrm{m}$. 


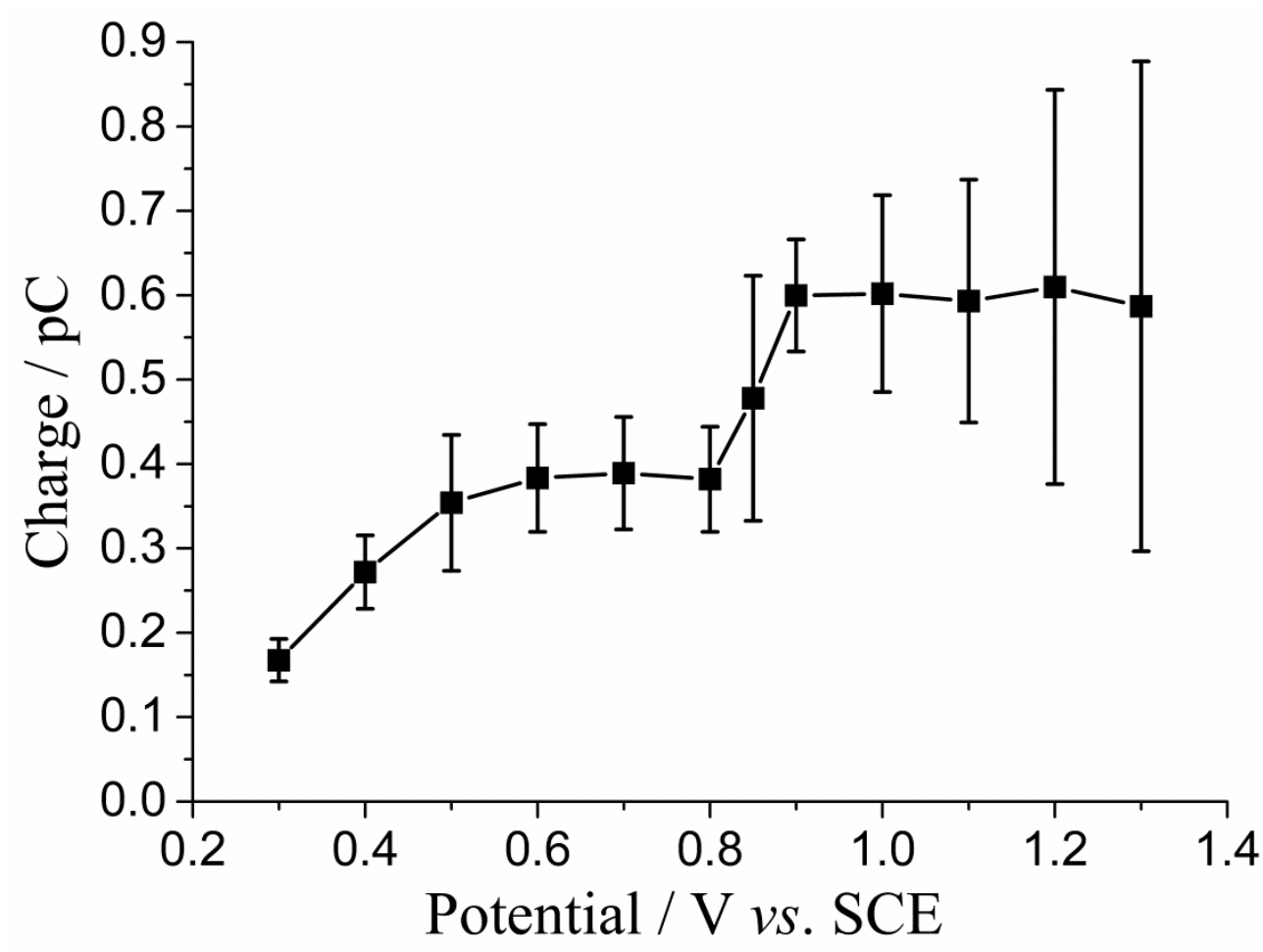

Figure 7. Potential variation of the mean charge passed per individual spike. The mean charge at each potential is generated from a log-normal distribution. The error of the mean charge is given by $S D / \sqrt{n^{\prime}}$, where SD is the standard deviation and $n$ ' is the number of spikes. ${ }^{25}$

In order to find the influence of potential on the impact experiments, chronoamperograms were recorded at different potentials, from +0.30 to $+1.30 \mathrm{~V}$, after the same amount of catecholalumina was added. The mean charge passed per individual spikes $(Q)$ was plotted against the potential, as shown in Figure 7. It can be seen that the amplitude of impact spikes is dependent on the potential applied. Overall the amount of charge observed around $+0.90 \mathrm{~V}$ and above is expected from the ensemble voltammetry discussed above where a two electron oxidation of $\mathrm{H}_{2} \mathrm{C}$ to $\mathrm{Q}$ is seen. However the entire plot resembles a "voltammogram" of two voltammetric waves, one near $+0.40 \mathrm{~V}$ as expected but the second at a much higher potential of $c a .+0.85 \mathrm{~V}$. This can be understood as follows.

When the potential is above $+0.90 \mathrm{~V}, Q$ remains constant at its maximum value of ca.1.1 pC, consistent with the two-electron oxidation of catechol being fully driven

$\mathrm{H}_{2} \mathrm{C}-2 \mathrm{e}^{-}-2 \mathrm{H}^{+} \quad \rightleftarrows \quad \mathrm{Q}$

At lower potentials between +0.60 and $+0.80 \mathrm{~V}$ it appears that only the process

$\mathrm{H}_{2} \mathrm{C}-\mathrm{e}^{-}-\mathrm{H}^{+} \rightleftarrows \mathrm{SH}$ 
is observed since approximately half the charge is passed. The onset potential of this process is similar to that seen in the ensemble voltammetry. The contrast between the impact and ensemble voltammetry likely lies in the mechanism by which $\mathrm{H}_{2} \mathrm{C}$ is converted into Q. As noted above this involves the following rate determining step ${ }^{19}$

$2 \mathrm{SH} \rightarrow \quad \mathrm{Q}+\mathrm{H}_{2} \mathrm{C}$

so that a DISP type mechanism is followed. The measurement timescale for ensemble voltammetry is relatively slow (seconds) so that there is ample time for the disproportionation of SH to occur on the voltammetry timescale and hence for a full two electron reaction to be seen around $+0.40 \mathrm{~V}$ and at more positive potentials. In contrast during impact coulometry the duration of the impacts is limited to around ca. $10-100 \mathrm{~ms}$. It seems therefore that the disproportionation kinetic step is outrun during impacts and so for potentials around $+0.40 \mathrm{~V}$ the reaction is limited at the one electron stage. In order for a second electron to be transferred the reactant must be measured to $c a$. $+0.85 \mathrm{~V}$ to drive the reaction

$\mathrm{SH}-\mathrm{e}^{-}-\mathrm{H}^{+} \quad \rightleftarrows \quad \mathrm{Q}$

via electron transfer from SH to the electrode rather than via disproportionation followed by electron transfer from $\mathrm{H}_{2} \mathrm{C}$. Note therefore that a change of mechanism is seen between the impact and ensemble voltammetry. The mechanisms and contrasts are summarised in Scheme 4. 


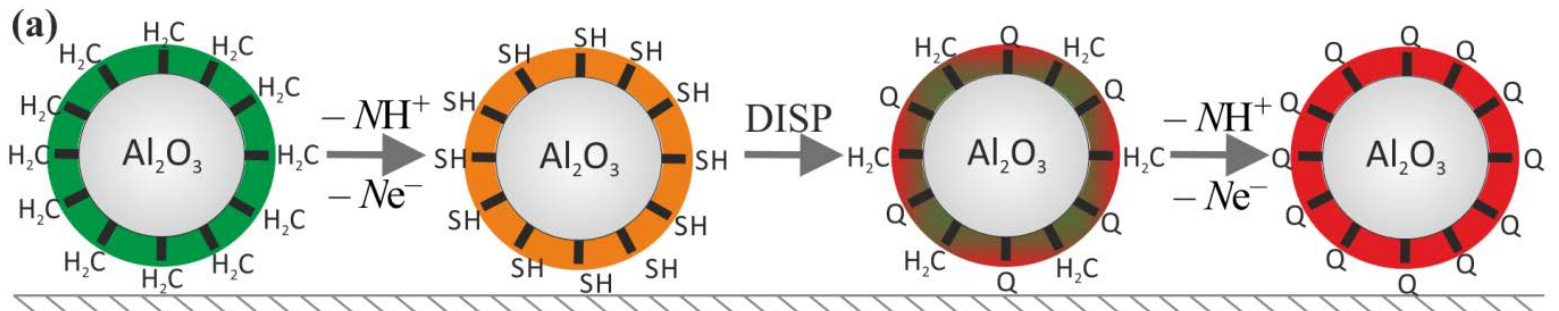

(b)

(c)

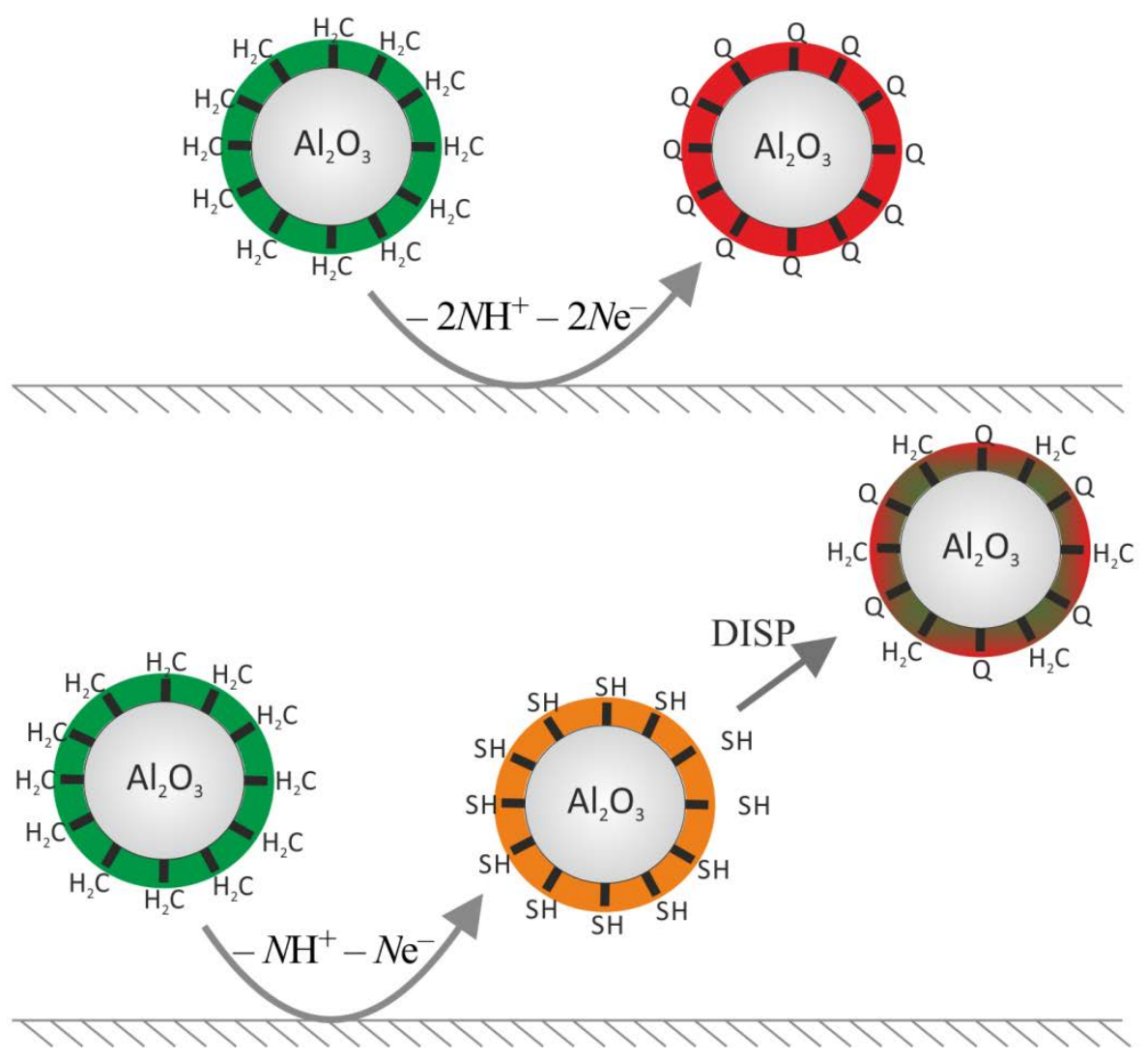

Scheme 4. The different mechanisms corresponding to (a) ensemble voltammetry when the potential is at $+0.40 \mathrm{~V}$, (b) impact voltammetry when the potential is above $+0.90 \mathrm{~V}$, and (c) impact voltammetry when the potential is above $+0.40 \mathrm{~V}$. $N$ is number of catechol molecules adsorbed per alumina particle.

\subsection{Modelling Individual Current Spikes}

Following the model of charge diffusion over the surface of a sphere (Scheme 2), ${ }^{17}$ the individual impact spikes can be used to determine the diffusion coefficient $(D)$ of charge transfer between catechol molecules over the surface of alumina particles. The coverage $\left(\Gamma_{i}\right)$ of catechol on the surface of alumina can also be derived. Individual impact spikes from chronoamperograms at $+0.90 \mathrm{~V}$ were picked for baseline correction and analysis. Then the decaying part of the transient was analysed (Figure 8 inset, red line). Figure 8 illustrates the fitting of the experimental data (in red) in order to fit 
the curvature of the theoretical data (in black) (note the log-log scale). This fitting was repeated for numerous individual impact spikes to obtain the mean value of $D$ and $\Gamma_{\mathrm{i}}$.

Given the relation between $I$ and $N u$, using Eq. 7 and 11 together with the "working curve" shown in Figure 1, we find that $D=(2.5 \pm 0.5) \times 10^{-6} \mathrm{~cm}^{2} \mathrm{~s}^{-1}$ and $\Gamma_{\mathrm{i}}=(5.9 \pm 1.9) \times 10^{-10} \mathrm{~mol} \mathrm{~cm}^{-2}$. The value of $\Gamma_{\mathrm{i}}$ is consistent with the value measured from full integration of the spike charge.

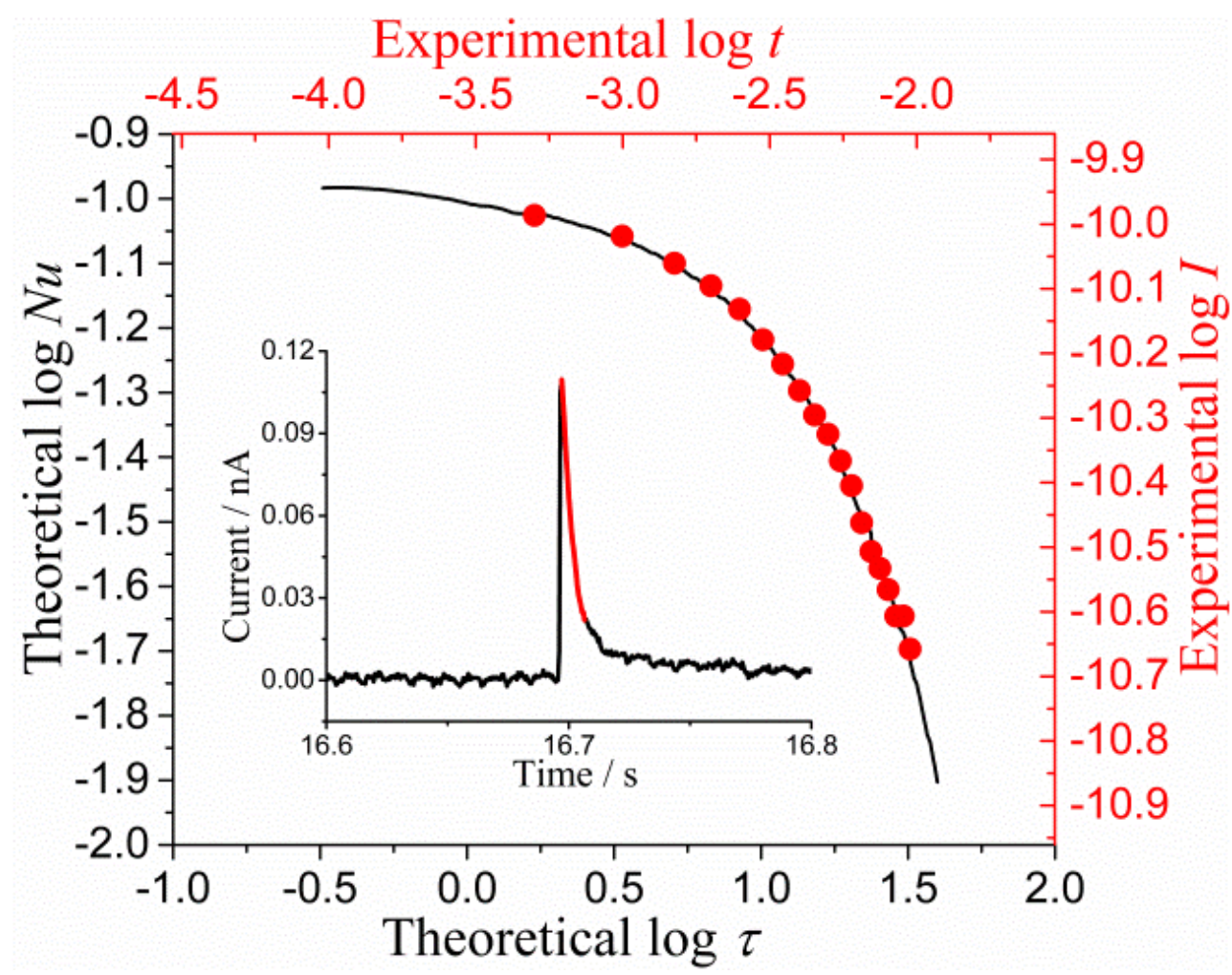

Figure 8. Comparison of experimental (in red) and theoretical (in black) data. The scale of horizontal and vertical axes is 3.0 and 1.1, respectively, for both experimental and theoretical data. Inset: chronoamperometry of the individual spike with baseline corrected. The transient in red is chosen for analysis, the logarithm of which corresponds to the red dots.

\section{CONCLUSIONS}

A new electrochemical method has been developed to use particle impacts to quantify the adsorption of redox active species on the surface of insulating particles. The amplitude of impact spikes has been found to vary with the potential applied to the electrode. At high potential, the oxidation of catechol can be fully driven to two-electron process; while at low potential, only one-electron oxidation occurs since the kinetics of the disproportionation reaction that operates in ensemble voltammetry are "out run”. 
Using the theory of charge diffusion over the surface of a sphere, individual impact spikes were analysed to give the diffusion coefficient of charge transfer between catechol molecules over the surface of alumina as $(2.5 \pm 0.5) \times 10^{-6} \mathrm{~cm}^{2} \mathrm{~s}^{-1}$. The coverage of catechol on the alumina surface was found to be $(5.9 \pm 1.9) \times 10^{-10} \mathrm{~mol} \mathrm{~cm}^{-2}$. This value is close in agreement with an estimation made assuming that catechol molecules are close-packed in a monolayer over the surface of the alumina.

\section{REFERENCES}

(1) Zhou, Y.-G.; Rees, N. V.; Compton, R. G. The Electrochemical Detection and Characterization of Silver Nanoparticles in Aqueous Solution. Angew. Chem. Int. Ed. 2011, 50, 4219-4221.

(2) Zhou, Y.-G.; Rees, N. V.; Pillay, J.; Tshikhudo, R.; Vilakazi, S.; Compton, R. G. Gold Nanoparticles Show Electroactivity: Counting and Sorting Nanoparticles Upon Impact with Electrodes. Chem. Commun. 2012, 48, 224-226.

(3) Stuart, E. J. E.; Zhou, Y.-G.; Rees, N. V.; Compton, R. G. Determining Unknown Concentrations of Nanoparticles: The Particle-Impact Electrochemistry of Nickel and Silver. RSC Adv. 2012, 2, 68796884.

(4) Zhou, Y.-G.; Haddou, B.; Rees, N. V.; Compton, R. G. The Charge Transfer Kinetics of the Oxidation of Silver and Nickel Nanoparticles via Particle-Electrode Impact Electrochemistry. Phys. Chem. Chem. Phys. 2012, 14, 14354-14357.

(5) Haddou, B.; Rees, N. V.; Compton, R. G. Nanoparticle-Electrode Impacts: The Oxidation of Copper Nanoparticles Has Slow Kinetics. Phys. Chem. Chem. Phys. 2012, 14, 13612-13617.

(6) Tschulik, K.; Haddou, B.; Omanović, D.; Rees, N.; Compton, R. Coulometric Sizing of Nanoparticles: Cathodic and Anodic Impact Experiments Open Two Independent Routes to Electrochemical Sizing of $\mathrm{Fe}_{3} \mathrm{O}_{4}$ Nanoparticles. Nano Res. 2013, 6, 836-841.

(7) Quinn, B. M.; van 't Hof, P. G.; Lemay, S. G. Time-Resolved Electrochemical Detection of Discrete Adsorption Events. J. Am. Chem. Soc. 2004, 126, 8360-8361.

(8) Banks, C. E.; Rees, N. V.; Compton, R. G. Sonoelectrochemistry Understood via Nanosecond Voltammetry: Sono-Emulsions and the Measurement of the Potential of Zero Charge of a Solid Electrode. J. Phys. Chem. B 2002, 106, 5810-5813.

(9) Rees, N. V.; Banks, C. E.; Compton, R. G. Ultrafast Chronoamperometry of Acoustically Agitated Solid Particulate Suspensions: Nonfaradaic and Faradaic Processes at a Polycrystalline Gold Electrode. J. Phys. Chem. B 2004, 108, 18391-18394.

(10) Scholz, F.; Hellberg, D.; Harnisch, F.; Hummel, A.; Hasse, U. Detection of the Adhesion Events of Dispersed Single Montmorillonite Particles at a Static Mercury Drop Electrode. Electrochem. Commun. 2004, 6, 929-933.

(11) Hellberg, D.; Scholz, F.; Schauer, F.; Weitschies, W. Bursting and Spreading of Liposomes on the Surface of a Static Mercury Drop Electrode. Electrochem. Commun. 2002, 4, 305-309.

(12) Hellberg, D.; Scholz, F.; Schubert, F.; Lovrić, M.; Omanović, D.; Hernández, V. A.; Thede, R. Kinetics of Liposome Adhesion on a Mercury Electrode. J. Phys. Chem. B 2005, 109, 14715-14726.

(13) Agmo Hernández, V.; Scholz, F. Kinetics of the Adhesion of Dmpc Liposomes on a Mercury Electrode. Effect of Lamellarity, Phase Composition, Size and Curvature of Liposomes, and Presence of the Pore Forming Peptide Mastoparan X†. Langmuir 2006, 22, 10723-10731.

(14) Poon, J.; Lin, Q.; Batchelor-McAuley, C.; Salter, C.; Johnston, C.; Compton, R. G. Altered Electrochemistry at Graphene- or Alumina-Modified Electrodes: Catalysis vs Electrocatalysis in Multistep Electrode Processes. J. Phys. Chem. C 2015, 119, 13777-13784.

(15) Zhou, Y.-G.; Rees, N. V.; Compton, R. G. The Electrochemical Detection of Tagged Nanoparticlesvia Particle-Electrode Collisions: Nanoelectroanalysis Beyond Immobilisation. Chem. Commun. 2012, 48, 2510-2512. 
(16) Rees, N. V.; Zhou, Y.-G.; Compton, R. G. The Non-Destructive Sizing of Nanoparticles via Particle-Electrode Collisions: Tag-Redox Coulometry (TRC). Chem. Phys. Lett. 2012, 525-526, 6971.

(17) Thompson, M.; Wildgoose, G. G.; Compton, R. G. The Theory of Non-Cottrellian Diffusion on the Surface of a Sphere or Truncated Sphere. ChemPhysChem 2006, 7, 1328-1336.

(18) Amatore, C.; Bouret, Y.; Maisonhaute, E.; Goldsmith, J. I.; Abruña, H. D. Precise Adjustment of Nanometric-Scale Diffusion Layers within a Redox Dendrimer Molecule by Ultrafast Cyclic Voltammetry: An Electrochemical Nanometric Microtome. Chem. - Eur. J. 2001, 7, 2206-2226.

(19) Lin, Q.; Li, Q.; Batchelor-McAuley, C.; Compton, R. G. Two-Electron, Two-Proton Oxidation of Catechol: Kinetics and Apparent Catalysis. J. Phys. Chem. C 2015, 119, 1489-1495.

(20) Xiao, X.; Bard, A. J. Observing Single Nanoparticle Collisions at an Ultramicroelectrode by Electrocatalytic Amplification. J. Am. Chem. Soc. 2007, 129, 9610-9612.

(21) Cheng, W.; Compton, R. G. Investigation of Single-Drug-Encapsulating Liposomes Using the Nano-Impact Method. Angew. Chem. Int. Ed. 2014, 53, 13928-13930.

(22) Barnes, E. O.; Zhou, Y.-G.; Rees, N. V.; Compton, R. G. The Effect of Near Wall Hindered Diffusion on Nanoparticle-Electrode Impacts: A Computational Model. J. Electroanal. Chem. 2013, 691, 28-34.

(23) Kätelhön, E.; Compton, R. G. Understanding Nano-Impacts: Impact Times and Near-Wall Hindered Diffusion. Chem. Sci. 2014, 5, 4592-4598.

(24) Ellison, J.; Tschulik, K.; Stuart, E. J. E.; Jurkschat, K.; Omanović, D.; Uhlemann, M.; Crossley, A.; Compton, R. G. Get More out of Your Data: A New Approach to Agglomeration and Aggregation Studies Using Nanoparticle Impact Experiments. ChemistryOpen 2013, 2, 69-75.

(25) Stuart, E. J. E.; Tschulik, K.; Batchelor-McAuley, C.; Compton, R. G. Electrochemical Observation of Single Collision Events: Fullerene Nanoparticles. ACS Nano 2014, 8, 7648-7654.

\section{TOC GRAPH}

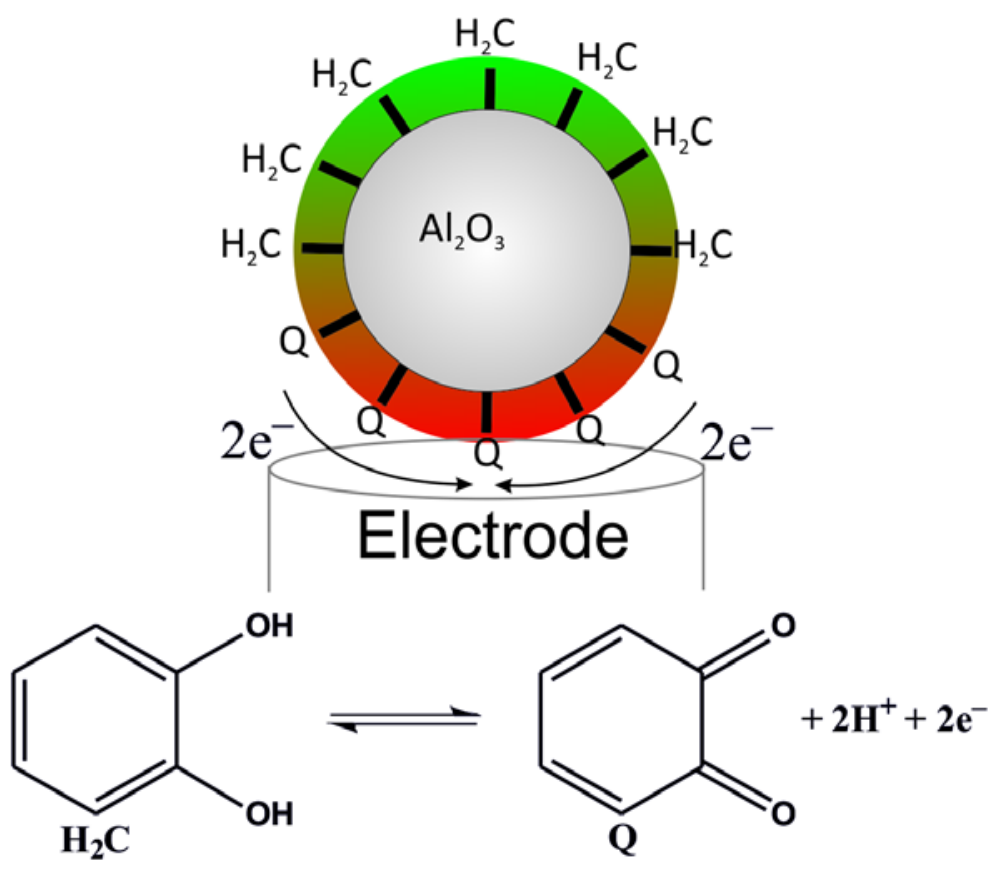

